\title{
Wafer scale synthesis of organic semiconductor nanosheets for van der Waals heterojunction devices
}

\author{
Sirri Batuhan Kalkan $\mathbb{D}^{1,4}$, Emad Najafidehaghani ${ }^{2,4}$, Ziyang Gan ${ }^{2}$, Fabian Alexander Christian Apfelbeck', Uwe Hübner ${ }^{3}$, \\ Antony George $\mathbb{( \mathbb { D }}^{2}$, Andrey Turchanin $\mathbb{1 0}^{2 \otimes}$ and Bert Nickel $\mathbb{B}^{1 凶}$
}

Organic semiconductors (OSC) are widely used for consumer electronic products owing to their attractive properties such as flexibility and low production cost. Atomically thin transition metal dichalcogenides (TMDs) are another class of emerging materials with superior electronic and optical properties. Integrating them into van der Waals (vdW) heterostructures provides an opportunity to harness the advantages of both material systems. However, building such heterojunctions by conventional physical vapor deposition (PVD) of OSCs is challenging, since the growth is disrupted due to limited diffusion of the molecules on the TMD surface. Here we report wafer-scale (3-inch) fabrication of transferable OSC nanosheets with thickness down to $15 \mathrm{~nm}$, which enable the realization of heterojunction devices. By controlled dissolution of a poly(acrylic acid) film, on which the OSC films were grown by PVD, they can be released and transferred onto arbitrary substrates. OSC crystal quality and optical anisotropy are preserved during the transfer process. By transferring OSC nanosheets (p-type) onto prefabricated electrodes and TMD monolayers (n-type), we fabricate and characterize various electronic devices including unipolar, ambipolar and antiambipolar field-effect transistors. Such $\mathrm{vdW} p$-n heterojunction devices open up a wide range of possible applications ranging from ultrafast photodetectors to conformal electronics.

npj 2D Materials and Applications (2021)5:92; https://doi.org/10.1038/s41699-021-00270-9

\section{INTRODUCTION}

Heterojunctions based on organic semiconductors (OSCs) and monolayer transition metal dichalcogenides (ML TMDs), exploiting the advantages of both material systems, attract interest in the engineering of electronic, photonic and optoelectronic devices ${ }^{1-3}$. OSC exhibit excellent electronic properties which can outperform or act complementary to inorganic semiconductors ${ }^{4-6}$. OSC based electronic and optoelectronic devices such as organic light-emitting diodes (OLED) ${ }^{7}$ are already used as constituents of several consumer products widely, especially in displays of television sets and mobile phones. ML TMDs are known for their superior electronic transport properties and have been identified as promising candidates for ultrathin device technologies ${ }^{8,9}$. The combination of OSCs and highperformance ML TMDs provides novel opportunities for complementary metal-oxide-semiconductor (CMOS) technology ${ }^{10,11}$. However, integration of OSCs and TMDs into the heterojunction devices via physical vapor deposition (PVD) of OSCs onto the TMD surfaces is challenging, since the low diffusion and ordering of the organic molecules limits functional properties of the formed OSC films ${ }^{12,13}$. Here we report a methodology for fabrication of highly crystalline ultrathin (down to $15 \mathrm{~nm}$ ) OSC nanosheets with lateral dimensions up to 3-inch and their transfer onto arbitrary substrates. We employ this methodology for the assembly of van der Waals (vdW) heterostructures with two-dimensional (2D) materials. As an example for 2D materials, we chose ML TMDs. In brief, water-soluble polyacrylic acid (PAA) thin films on silicon wafers were used as growth substrates to form highly crystalline OSC films of pentacene or dinaphtho[2,3-b:2',3'-f]thieno[3,2-b]thiophene (DNTT) via PVD. We show that these films can be controllably released as mechanically stable nanosheets from the growth substrate by the dissolution of the water-soluble PAA substrate and transferred onto arbitrary substrates. We apply various characterization techniques such as atomic force microscopy (AFM), X-ray diffraction and confocal microscopy to demonstrate the high crystallinity and therewith optical anisotropy of the OSC nanosheets before and after transfer. We use the OSC nanosheets to fabricate field-effect transistors (FETs) and to study their performance. To this end, the nanosheets were transferred onto prefabricated electrodes to realize wafer-scale bottom-contact FET arrays and their properties were compared with OSC films directly grown on the wafer by PVD. Besides that, the p-type OSC nanosheets were transferred onto prefabricated n-type $\mathrm{MoS}_{2}$ device structures to realize high-performance ambipolar and antiambipolar FETs.

\section{RESULTS AND DISCUSSION}

\section{Release and transfer of organic nanosheets}

First, we describe the transfer method. We spin coat a watersoluble PAA thin film on an oxygen plasma-treated 3-inch Si wafer. The PAA layer acts as the growth substrate for PVD of highly ordered pentacene or DNTT films with thicknesses ranging from 15 to $50 \mathrm{~nm}$. We found out that a controlled release of the OSC films is possible by a water-assisted transfer technique, see Fig. 1. We place a Si wafer with the OSC film next to the water droplet, see Fig. 1a and Supplementary Fig. 1, and establish a contact between the wafer and the droplet, see Fig. 1b. The surface tension difference between the untreated glass plate and the plasma-treated $\mathrm{Si}$ wafer drives the water towards the $\mathrm{Si}^{14}$; the water intrudes between the substrate and the OSC film, which results in its release from the substrate and formation of a freestanding OSC nanosheet. Due to the hydrophobic nature of the $\mathrm{OSC}^{15}$, the released nanosheet floats on the water meniscus.

\footnotetext{
${ }^{1}$ Faculty of Physics and CeNS, Ludwig-Maximilians-Universität, Geschwister-Scholl-Platz 1, 80539 Munich, Germany. ${ }^{2}$ Institute of Physical Chemistry and Abbe Center of Photonics, Friedrich Schiller University Jena, Lessingstr. 10, 07743 Jena, Germany. ${ }^{3}$ Leibniz Institute of Photonic Technology (IPHT), Albert-Einstein-Str. 9, 07745 Jena, Germany. ${ }^{4}$ These authors contributed equally: Sirri Batuhan Kalkan, Emad Najafidehaghani. ${ }^{凶}$ email: andrey.turchanin@uni-jena.de; nickel@lmu.de
} 


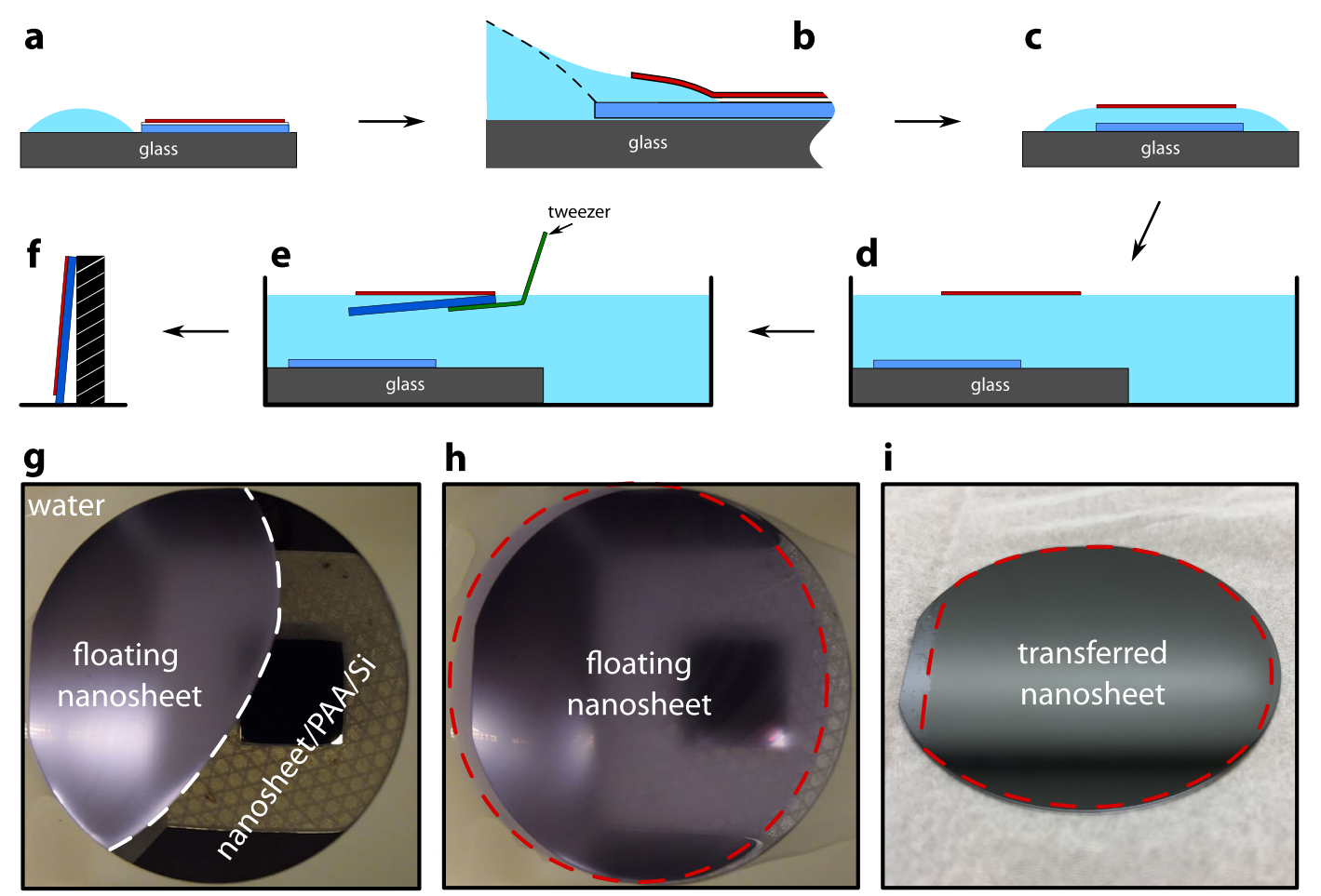

Fig. 1 Schemes and photographs of the water meniscus assisted transfer process. a A Si wafer with an OSC film on a PAA interlayer is placed on a glass plate next to a water droplet, the water contact angle is ca. $50^{\circ}$. b After the Si wafer contacted the droplet, PAA starts to dissolve and the OSC film is partially released. c After some time, the OSC film is detached and floats on the water layer as a mechanically stable nanosheet. $\mathbf{d}$ The Si wafer with the floating nanosheet on the glass plate is immersed into a beaker filled with water. e The floating OSC nanosheet is moved to the target substrate. $\mathbf{f}$ The target substrate with OSC is removed from the beaker, and placed almost vertically inclined for drying. The OSC nanosheet adheres homogeneously to the substrate. g Photograph of the PAA dissolution step as illustrated in (b). A partially detached pentacene nanosheet (thickness of $50 \mathrm{~nm}$ and diameter of 3-inch) can be seen floating on the water meniscus. In the right side of the wafer, it can be seen that nanosheet is still attached to the wafer. The floating part of the nanosheet appears hazy. $\mathbf{h}$ The pentacene nanosheet floating on water after complete dissolution of PAA. The pentacene nanosheet indicated as red-dashed circle. $\mathbf{i}$ Photograph of the target wafer with transferred pentacene nanosheet.

Although the rest of the OSC film is still sticking to the Si wafer at one side, it remains intact without rupture or cracking. A photograph of an intermediate state of the release of a $50 \mathrm{~nm}$ thick pentacene film from a 3-inch Si wafer is shown in Fig. 1g. Finally, by adding more water, the PAA dissolves completely and the whole mechanically stable OSC nanosheet floats on the water surface, Fig. 1c. A photograph of such a $50 \mathrm{~nm}$ thick pentacene nanosheet is shown in Fig. $1 \mathrm{~h}$. After the organic film is released from the PAA template, the transfer of the OSC onto the target substrate resembles other meniscus-based transfer schemes for all-organic heterojunctions, which instead require soluble molecules $^{16-18}$. Further details on the PAA dissolution and a time-lapse video of the process are provided in Supplementary Notes 1 and in Supplementary Video 1. Next, the glass support with the floating OSC nanosheet on the wafer is immersed into a water bath (Fig. 1d) and the floating OSC nanosheet is taken by the target substrate, Fig. 1e. Note that transfer of the nanosheet is particularly favorable on hydrophilic target substrates. However, the transfer onto hydrophobic target substrates such as Si wafers after cleanroom processing is also possible. To this end, the edge of the target Si wafer needs to establish a physical contact with the floating OSC nanosheet to enable vdW adhesion as shown schematically in Fig. 1e. Once the adhesion is established, the target wafer with the OSC nanosheet is taken out of the water and dried in air. We found out that positioning the wafer almost vertically allows excess water to drain out easily and dry faster, Fig. 1f. A $50 \mathrm{~nm}$ pentacene nanosheet transferred in this way on a 2-inch wafer is shown in Fig. 1i.

\section{Structural and optical characterization of transferred organic nanosheets}

Morphology and crystallinity of the OSC nanosheets were characterized before and after transfer by AFM and X-ray diffraction. For $50 \mathrm{~nm}$ pentacene and DNTT nanosheets, PAA promotes the formation of large crystalline grains with lateral grain size dimensions of $\sim 6 \mu \mathrm{m}$ and $\sim 3 \mu \mathrm{m}$, respectively (see Fig. $2 \mathrm{a}, \mathrm{d}$ ). These grains are much larger than those obtained by the direct growth on oxide surfaces such as $\mathrm{SiO}_{2}$ and $\mathrm{Al}_{2} \mathrm{O}_{3}$, or on TMDs, see Supplementary Fig. 3 for a comparison. After transfer, the grain morphology of the OSC nanosheets is preserved as verified by AFM, see Fig. 2b, e and Supplementary Fig. 4. X-ray diffraction further confirms that PAA promotes OSC nanosheet growth in a single crystallographic phase, i.e., before transfer, there is only one series of Bragg peaks for both materials (see black curves in Fig. 2c, f). The Bragg reflections of pentacene change after transfer (see red curve in Fig. 2c). Pentacene is known to exhibit different polymorphs such as thin film and Campbell bulk phase which can be converted in response to stress ${ }^{19}$. Apparently, the transfer induces a stress sufficient to convert some fraction of the thin-film phase into Campbell bulk phase ${ }^{20}$. Such stress might originate from the curvature of the water meniscus during the release of the OSC nanosheet. Stress-induced phase transitions have not been reported for DNTT. In agreement, the crystallographic phase of the DNTT nanosheet is preserved after transfer.

It is well-known that the optical reflection of pentacene grains is strongly polarization dependent for some specific wavelengths, a phenomenon called Davydov splitting ${ }^{21}$. In order to probe for 

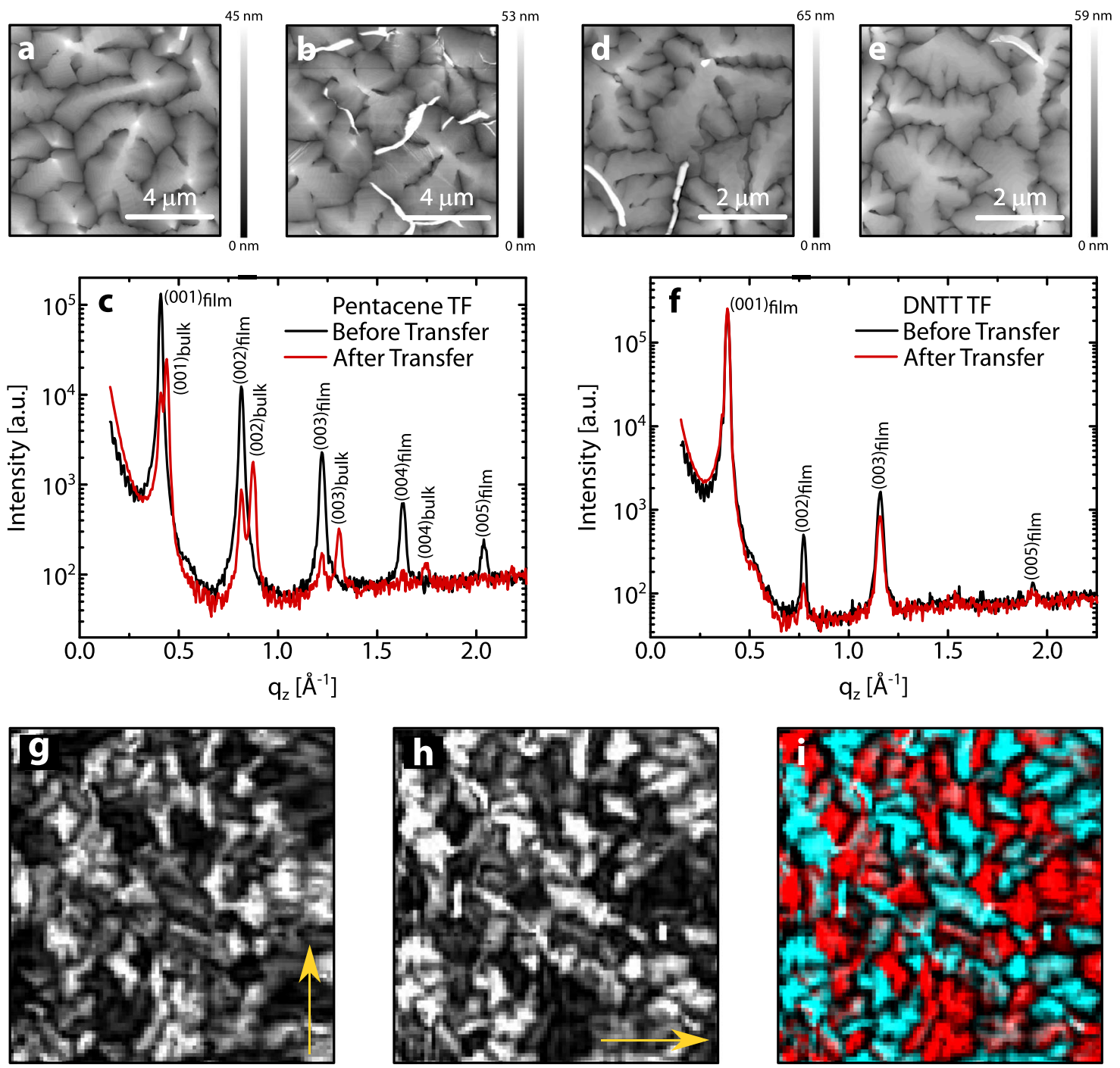

Fig. 2 Structural and optical characterization of $50 \mathrm{~nm}$ OSC nanosheets before and after transfer. AFM micrographs of pentacene nanosheet (a) before and (b) after transfer. (c) X-ray diffraction measurements of pentacene nanosheet before and after transfer, black curve and red curve, respectively. AFM micrographs of DNTT nanosheet (d) before and (e) after transfer. $\mathbf{f}$ X-ray diffraction measurements of DNTT nanosheet before and after transfer, black and red curve, respectively. g Optical reflection maps of the transferred pentacene nanosheet recorded by mapping an area of approx. $25 \mu \mathrm{m} \times 25 \mu \mathrm{m}$ with a polarized laser scanning confocal microscope at $635 \mathrm{~nm}$. The yellow arrow indicates the polarization as vertical in (g), and as horizontal polarization in (h). i False color superimposed image combining (g) and (h).

Davydov splitting, we raster scan the transferred pentacene nanosheet in confocal geometry with a linear polarized laser beam $(635 \mathrm{~nm})$ (see Supplementary Notes 4). Indeed, we found out that the reflection of individual grains depends on the polarization direction of the laser, which is indicated by the yellow arrow in Fig. $2 \mathrm{~g}$, h. Each grain reflects only one of the two polarization states efficiently, as verified by a false-colour superposition map in Fig. $2 \mathrm{i}$. This observation confirms that the transferred pentacene nanosheets preserves their specific optical properties.

\section{Transport characteristics of transferred organic nanosheets}

Next, the electronic transport properties of transferred OSC nanosheets were investigated in the inverted coplanar fieldeffect geometry, i.e., bottom-contact and bottom-gate FETs, as shown in Fig. 3a (see also Supplementary Fig. 5). A highly doped Si substrate is used as a global bottom-gate electrode and an ALD grown $33 \mathrm{~nm}$ alumina $\left(\mathrm{Al}_{2} \mathrm{O}_{3}\right)$ film serves as the gate dielectric. $A$ self-assembled monolayer (SAM) of n-tetradecylphosphonic acid was used to passivate the alumina film ${ }^{6}$. Bottom-contacts were defined by shadow masks, which we use to evaporate a structured
$\mathrm{Ti}$ adhesion layer and $\mathrm{Au}$ contacts. Finally, a $50 \mathrm{~nm}$ DNTT nanosheet is transferred on the wafer. The device characterization is shown in Fig. 3b. The DNTT device exhibits an on/off ratio of up to $10^{5}$ at $V_{D}=-5.0 \mathrm{~V}$, a threshold voltage of $-0.63 \mathrm{~V}$, and a little hysteresis of $217 \mathrm{mV}$ (see Supplementary Fig. 6 for further transistor characteristics). The extracted mobility for DNTT is $0.16 \mathrm{~cm}^{2} \mathrm{~V}^{-1} \mathrm{~s}^{-1}$ and the subthreshold swing is $289 \mathrm{mV}$ per decade. A further improvement of the extracted OSC mobility might be possible by bottom-contact modification ${ }^{22}$. These values show that the OSC/gate dielectric junction is structurally and energetically well-ordered after the nanosheet transfer, i.e. we see only mild signatures of the charge traps that typically lower device performance in OSC bottom-contact devices. In addition, transferred nanosheet FETs with top-contacts show similar transfer characteristics as bottom-contact devices suggesting that the well-known $\mathrm{Au}$ bottom-contact problems were resolved by transfer (Supplementary Fig. 7).

Next, we compare the device with a transferred nanosheet with a similar device fabricated via direct PVD of the DNTT. This device yields drain currents, which are more than one order of magnitude 


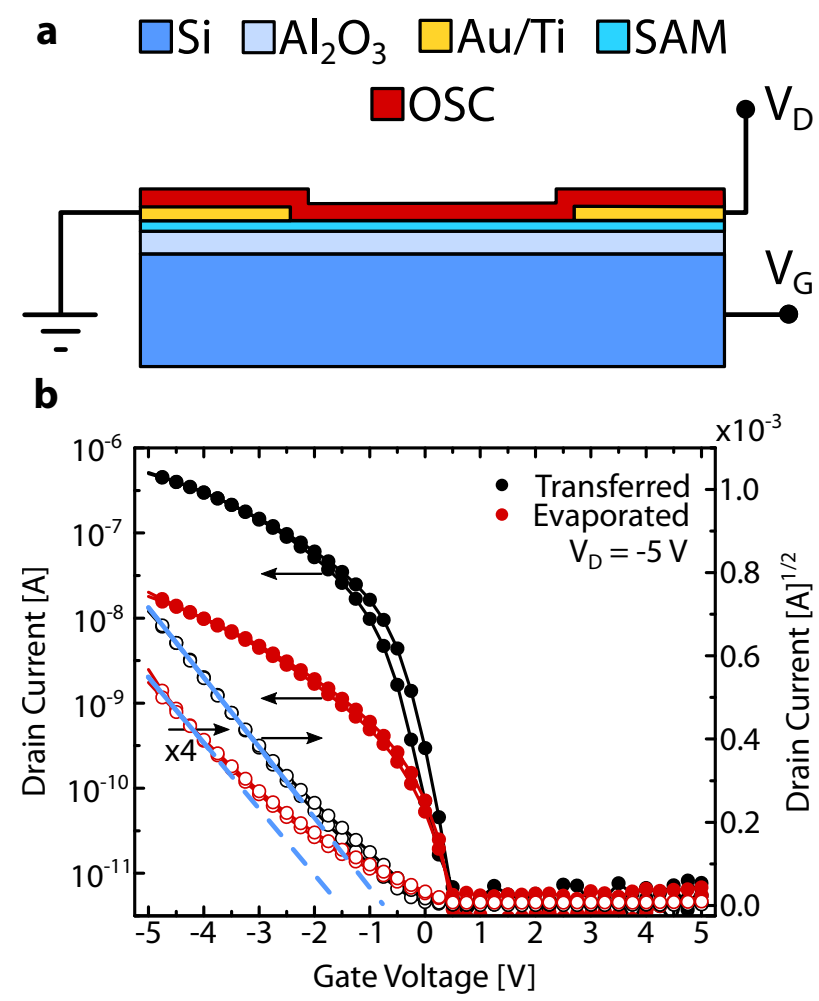

Fig. 3 Electrical characterization of DNTT FETs. a Schematic of OSC nanosheet FET device. The Si device substrate has a $33 \mathrm{~nm}$ alumina gate dielectric layer modified with a n-tetradecylphosphonic acid SAM and Au/Ti source-drain electrodes. The heavily doped $\mathrm{Si}$ base functions as a gate electrode. A $50 \mathrm{~nm}$ thick DNTT nanosheet is transferred on to the device substrate which functions as the FET channel. For comparison another device is fabricated by direct PVD of a $50 \mathrm{~nm}$ thick DNTT layer onto a similar device structure. b Drain current $\left(I_{D}\right)$ as a function of gate voltage $\left(V_{\mathrm{G}}\right)$ of transferred DNTT nanosheet and evaporated DNTT film, shown as black and red curves, respectively. Filled and hollow circles correspond to logarithmic and square root axis, respectively. The drain bias voltage is $V_{D}=-5.0 \mathrm{~V}$. See Supplementary Notes 5 for details. Blue lines indicate the linear behavior in square root plot.

lower in comparison to the nanosheet device. The difference in performance can be further quantified by square root plots of the drain current; here the devices should exhibit an extended linear characteristic curve according to Shockley theory ${ }^{23}$. Indeed, the transferred device shows a linear region allowing for the extraction of the mobility, while the PVD device shows pronounced nonideal super-linear behavior, i.e., increasing slope with increasing gate voltage, which prevents the mobility extraction ${ }^{24}$. Eye inspection of Fig. $3 b$ reveals that the extrapolation of the threshold voltage (blue dashed line) is only valid for the four highest data points, which is an indication of super-linear behavior ${ }^{24}$. For the transferred device, the extrapolation is valid for a much larger voltage region, shown as the blue line in Fig. $3 \mathrm{~b}$ as well, an indication of close to ideal behavior ${ }^{24}$. A well-known cause for pronounced nonideal behavior in bottom-contact devices is the structural disorder of the OSC/Au junction due to the inferior growth behavior of organic molecules on $\mathrm{Au}$ surfaces ${ }^{25}$. Our results show that such growth problems are prevented in the FET devices made of the transferred OSC nanosheets.

\section{Transport characteristics of van der Waals heterostructures}

Now, we demonstrate the vdW heterostructure devices made of OSC nanosheets and ML $\mathrm{MoS}_{2}$. We assemble vdW p-n heterojunction by transfer of the OSC nanosheets onto ML MoS nanosheets. We tested two different device configurations resulting in ambipolar and antiambipolar FETs, see Fig. 4a, c. To this end, high-quality ML MoS 2 single crystals ${ }^{26}$ were synthesized on thermally oxidized Si wafers by chemical vapor deposition (CVD) (see Supplementary Notes 6). The $\mathrm{MoS}_{2}$ crystals were transferred by a PMMA assisted transfer process ${ }^{27}$ and $\mathrm{Au} / \mathrm{Ti}$ source/drain electrodes were patterned. Next, a $50 \mathrm{~nm}$ DNTT nanosheet was transferred (see Supplementary Fig. 9a). Figure 4b shows the transfer curves of such a device under ambient conditions demonstrating the characteristic drain current V-shape of ambipolar FETs, see also Supplementary Figure 10. The current in the ambipolar region can be expressed as the sum of an electron and hole current ${ }^{28}$ :

$$
\left|I_{D}\right|=\frac{W C_{i}}{2 L}\left\{\mu_{e}\left(V_{G}-V_{T h, e}\right)^{2}+\mu_{h}\left(V_{D}-\left(V_{G}-V_{T h, h}\right)\right)^{2}\right\}
$$

The drain voltage $V_{D}$ affects the threshold voltage of one of the two currents, (cf. Eq. 1), here the hole current, and in turn the transfer curve shifts in response to the drain voltage, cf. Fig. 4b. Furthermore, the two asymptotic branches of the drain current allow us to model the saturation behavior of the ambipolar device to determine electron and hole mobilities $\mu_{\mathrm{e}}, \mu_{\mathrm{h}}$ and threshold voltages $V_{\text {th,e, }} V_{\text {th,h }}$. The DNTT/MoS 2 vdW heterojunction device shows rather balanced mobilities of $\mu_{\mathrm{h}}=0.18 \mathrm{~cm}^{2} \mathrm{~V}^{-1} \mathrm{~s}^{-1}$ and $\mu_{\mathrm{e}}=$ $0.25 \mathrm{~cm}^{2} \mathrm{~V}^{-1} \mathrm{~s}^{-1}$. So far, similar balanced mobilities have only been reported for organic single crystals placed on 2D materials ${ }^{10}$. Such balanced mobilities are important in applications where the device operates in bipolar or ambipolar mode, as needed for, e.g., inverters and oscillators ${ }^{29}$. We performed drain bias dependent ambipolar FET measurements to resolve the nature of the conducting channel. We find that both channels ( $p$ and $n$ ) can be switched off in the ambipolar FET geometry via the gate, a typical behavior for a semiconductor (Supplementary Fig. 10a, b).

The drain current dip in the transfer curve provides additional information about the subthreshold behavior of the device. According to Eq. 1, the ambipolar current drops to zero for drain voltages smaller than the electron and hole threshold voltage difference. Here, we measure a finite current of $I_{\mathrm{D}}=100 \mathrm{pA}$ for $V_{\mathrm{D}}= \pm 5.0 \mathrm{~V}$ in the minimum. This current can be explained by subthreshold behavior of ML MoS 2 and DNTT; i.e., we can model current in the minimum with a ML MoS 2 subthreshold swing of $2.31 \mathrm{~V}$ per decade, see Supplementary Notes 7 for further analysis. The subthreshold swing allows to estimate the density of ML MoS subthreshold traps to $N_{\mathrm{t}, \text { sub }}=9.16 \times 10^{12} \mathrm{eV}^{-1} \mathrm{~cm}^{-2}$ assuming a capacitance per area of $C_{i}=38.4 \mathrm{nF}$ per $\mathrm{cm}^{2}$. Similar values are found for DNTT.

The geometric design of the ambipolar device allows that current flows in the two junction materials without crossing the two materials. In order to enforce current flow across the heterojunction, we employ now the antiambipolar geometry, Fig. 4c. In this geometry, the electrodes are exclusively in contact with one material only. Here, the source is connected to a ML $\mathrm{MoS}_{2}$ and drain to a $50 \mathrm{~nm}$ pentacene nanosheet. The overlap region of the two materials, i.e., the $p-n$ junction, is $15 \mu \mathrm{m}$ along the channel length. Technically, this configuration was realized with help of an insulating SU-8 polymer coating on one contact, and suited placement of the second contact (see Fig. $4 \mathrm{c}$ and Supplementary Fig. 9b, 11). In this antiambipolar geometry, the drain current minimum of the ambipolar geometry changes to a drain current maximum. This peak in drain current is the fingerprint of the antiambipolar geometry, confirming that indeed a functional $p-n$ heterostructure junction formed between a $2 D$ material and a transferred OSC. The signal to noise ratio of the drain current maximum is up to $10^{3}$, cf. Fig. $4 \mathrm{~d}$, exceeding previously reported values for this materials combination ${ }^{12}$. 

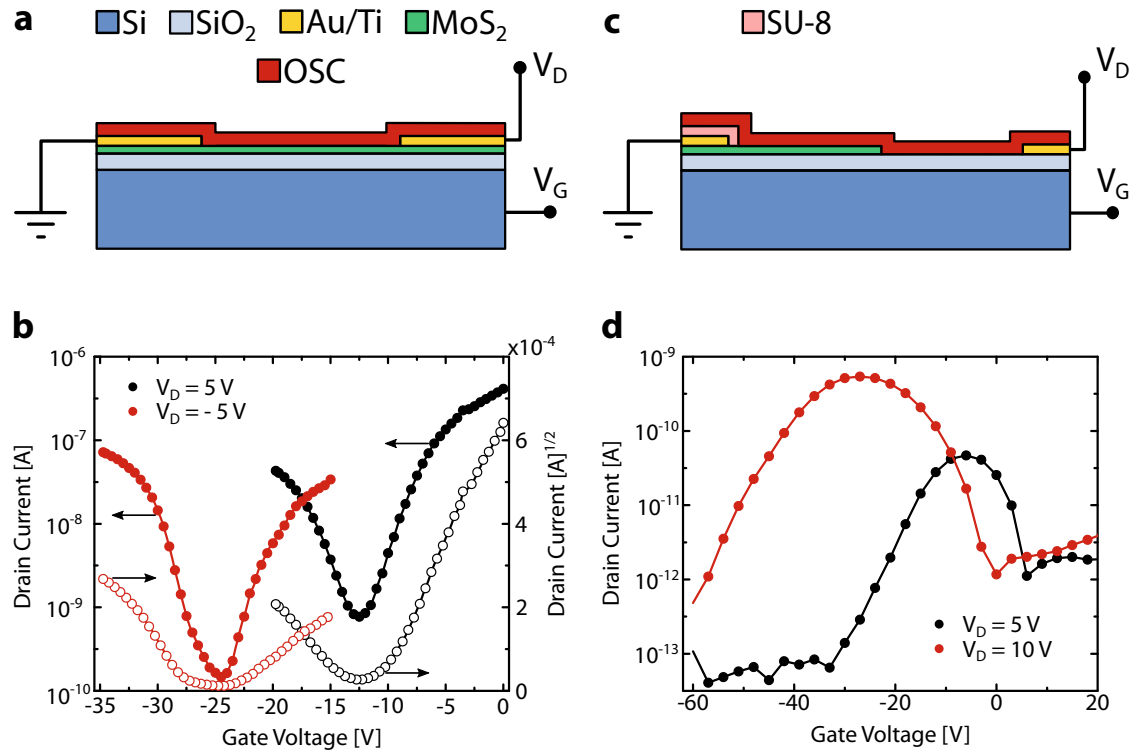

Fig. 4 Electrical characterization of ambipolar and antiambipolar devices fabricated by assembly of OSC nanosheets with ML MoS a $_{2}$ Schematic of the ambipolar FET device. As illustrated, the Au/Ti contacts are placed between the ML MoS 2 and the $50 \mathrm{~nm}$ thick DNTT nanosheet. Here the channel width and length are $10 \mu \mathrm{m}$. b Drain current as a function of gate voltage of the ambipolar FET device for two different drain voltages $(5.0 \mathrm{~V}$ and $-5.0 \mathrm{~V})$. Filled and hollow circles refer to logarithmic and square root axis, respectively. c Schematic diagram of antiambipolar FET device. The source and drain electrode are individually contacted to the $\mathrm{ML} \mathrm{MoS}_{2}$ and pentacene nanosheet, respectively. The pentacene/ML MoS 2 heterojunction is $15 \mu \mathrm{m}$ along the channel. $\mathbf{d}$ Drain current of the antiambipolar device as function of gate voltage for two different drain voltages $(5.0 \mathrm{~V}$ and $10.0 \mathrm{~V})$.

In summary, we have developed a method for wafer-scale (3inch) synthesis and transfer of organic semiconducting nanosheets with thickness down to $15 \mathrm{~nm}$, demonstrated for the example of DNTT and pentacene. The nanosheets can be transferred onto arbitrary substrates by a water-assisted transfer protocol. Unipolar devices fabricated via OSC transfer outperform prepared conventionally by PVD DNTT bottom-contact FETs by more than one order of magnitude in drain current. Fabrication of ambipolar and antiambipolar devices with OSC and ML MoS 2 was demonstrated and such devices reach the performance of heterojunction devices previously build from organic single crystals, outperforming also OSC films deposited by PVD directly on $\mathrm{MoS}_{2}$. Such vdW heterojunction devices have potential application in optoelectronics as ultrafast photodetectors or light-emitting devices. Further progress in this direction relies on the improvement of the subthreshold behavior. The OSC transfer method can be used to transfer OSC nanosheets to flexible or curved substrates for applications in large area photodetection (see Supplementary Fig. 14), biomedical sensing applications ${ }^{30}$, and mechanical sensing as in artificial skin devices ${ }^{31}$.

\section{METHODS}

\section{Organic semiconductor deposition}

Si wafer with $\mathrm{SiO}_{2}$ oxide surface were sonicated $5 \mathrm{~min}+10 \mathrm{~min}$ in acetone and in isopropanol then $10 \mathrm{~min} \mathrm{DI}$ at $60^{\circ} \mathrm{C}$. The $\mathrm{SiO}_{2}$ surface was activated by an $\mathrm{O}_{2}(10 \mathrm{sccm})$ plasma treatment at $50 \mathrm{~W}$ for $5 \mathrm{~min}$. An approx. $50 \mathrm{~nm}$ thick layer of PAA (volume fraction $2.5 \%$, filtered by $0.22 \mu \mathrm{m}$ pore-sized syringe filter) was spin-coated (60 s at $4000 \mathrm{rpm}$ ) onto the $\mathrm{Si}$ wafer. The wafers were immediately loaded into an ultra-high vacuum (UHV) chamber and up to $50 \mathrm{~nm}$ thick pentacene or DNTT films were evaporated at a base pressure of middle $10^{-8} \mathrm{mbar}$. The OSC films were evaporated with a rate of $0.1 \AA$ per $s$ at room temperature for pentacene and with a rate of $0.1 \AA$ per $s$ at $60^{\circ} \mathrm{C}$ for DNTT.

\section{AFM and X-ray measurements}

AFM images are recorded by a Bruker Dimensional Icon. The deposition rates and substrate temperatures for the nanosheets used in AFM measurements are $0.02 \AA$ per $s$ at room temperature and $0.1 \AA$ per $s$ at $60{ }^{\circ} \mathrm{C}$ for pentacene and DNTT respectively. For X-ray measurements an in house X-ray setup was used with Mo source and a monochromatic beam in reflection geometry. The deposition rates and substrate temperatures for the nanosheets used in X-ray measurements are $0.1 \AA$ per $s$ at room temperature for both pentacene and DNTT films, respectively.

\section{Device fabrication}

The shadow polyimide mask for patterning organic FETs was manufactured by CADiLAC Laser $\mathrm{GmbH}$. For vdW heterostructure FETs, $\mathrm{SiO}_{2}$ is used as gate dielectric and its thickness is $90 \mathrm{~nm}$ for ambipolar DNTT/ML $\mathrm{MoS}_{2}$ FET and $300 \mathrm{~nm}$ for antiambipolar pentacene/ML MoS 2 FET. The ambipolar FET was patterned by photolithography while the antiambipolar FET was patterned by e-beam lithography. OSC nanosheets for FETs were evaporated with $0.1 \AA$ per $s$ at room temperature for pentacene and $60^{\circ} \mathrm{C}$ for DNTT films.

\section{Electrical characterization}

A probe station in dark ambient conditions was used for organic FETs and DNTT/ML MoS 2 ambipolar FET where pentacene/ML MoS 2 antiambipolar FET was measured under vacuum.

\section{DATA AVAILABILITY}

The data that support the findings of this study are available from the corresponding author upon reasonable request.

Received: 14 July 2021; Accepted: 29 October 2021; Published online: 03 December 2021

\section{REFERENCES}

1. Jariwala, D., Marks, T. J. \& Hersam, M. C. Mixed-dimensional van der Waals heterostructures. Nat. Mater. 16, 170-181 (2017).

2. Gobbi, M., Orgiu, E. \& Samorì, P. When 2D materials meet molecules: opportunities and challenges of hybrid organic/inorganic van der Waals heterostructures. Adv. Mater. 30, 1706103 (2018).

3. Sun, J. et al. 2D-Organic hybrid heterostructures for optoelectronic applications. Adv. Mater. 31, 1803831 (2019). 
4. Horowitz, G. Organic field-effect transistors. Adv. Mater. 10, 365-377 (1998).

5. Yamamoto, T. \& Takimiya, K. Facile synthesis of highly $\pi$-extendedheteroarenes, dinaphtho $[2,3-b: 2 ', 3$ 3'-f] chalcogenopheno [3, 2-b] chalcogenophenes, and theirapplication to field-effect transistors. J. Am. Chem. Soc. 129, 2224-2225 (2007).

6. Klauk, H., Zschieschang, U., Pflaum, J. \& Halik, M. Ultralow-power organic complementary circuits. Nature 445, 745-748 (2007).

7. Leung, L. M. et al. A high-efficiency blue emitter for small molecule-based organic light-emitting diode. J. Am. Chem. Soc. 122, 5640-5641 (2000).

8. Radisavljevic, B. et al. Single-layer $\mathrm{MoS}_{2}$ transistors. Nat. Nanotechnol. 6, 147-150 (2011).

9. Larentis, S., Fallahazad, B. \& Tutuc, E. Field-effect transistors and intrinsic mobility in ultra-thin $\mathrm{MoSe}_{2}$ layers. Appl. Phys. Lett. 101, 223104 (2012).

10. $\mathrm{He}, \mathrm{X}$. et al. $\mathrm{MoS}_{2} /$ Rubrene van der Waals heterostructure: toward ambipolar fieldeffect transistors and inverter circuits. Small 13, 1602558 (2017).

11. Wang, Z., Huang, L. \& Chi, L. Organic semiconductor field-effect transistors based on organic-2D heterostructures. Front. Mat. 7, 295 (2020).

12. Jariwala, D. et al. Hybrid, gate-tunable, van der Waals $p-n$ heterojunctions from pentacene and $\mathrm{MoS}_{2}$. Nano Lett. 16, 497-503 (2016).

13. Kim, J.-K. et al. Trap-mediated electronic transport properties of gate-tunable pentacene/MoS 2 p-n heterojunction diodes. Sci. Rep. 6, 36775 (2016).

14. Eid, K., Panth, M. \& Sommers, A. The physics of water droplets on surfaces: exploring the effects of roughness and surface chemistry. Eur. J. Phys. 39, 025804 (2018).

15. Di, C.-a. et al. Effect of dielectric layers on device stability of pentacene-based field-effect transistors. Phys. Chem. Chem. Phys. 11, 7268-7273 (2009).

16. Li, H. et al. Organic Heterojunctions Formed by Interfacing Two Single Crystals from a Mixed Solution. J. Am. Chem. Soc. 141, 10007-10015 (2019).

17. Shi, Y. et al. Bottom-up growth of n-type monolayer molecular crystals on polymeric substrate for optoelectronic device applications. Nat. Commun. 9, 1-8 (2018).

18. Xiao, M. et al. Sub-5 $\mathrm{nm}$ single crystalline organic $\mathrm{p}-\mathrm{n}$ heterojunctions. Nat. Commun. 12, 1-7 (2021).

19. Murakami, Y. et al. Microstructural study of the polymorphic transformation in pentacene thin films. Phys. Rev. Lett. 103, 146102 (2009).

20. Westermeier, $C$. et al. Sub-micron phase coexistence in small-molecule organic thin films revealed by infrared nano-imaging. Nat. Commun. 5, 1-6 (2014).

21. Cocchi, C., Breuer, T., Witte, G. \& Draxl, C. Polarized absorbance and Davydov splitting in bulk and thin-film pentacene polymorphs. Phys. Chem. Chem. Phys. 20, 29724-29736 (2018).

22. Borchert, J. W. et al. Small contact resistance and high-frequency operation of flexible low-voltage inverted coplanar organic transistors. Nat. Commun. 10, 1-11 (2019).

23. Sze, S. M., Li, Y. \& Ng, K. K. Physics of Semiconductor Devices (John Wiley \& Sons, 2021).

24. Choi, H. H. et al. Critical assessment of charge mobility extraction in FETs. Nat. Mater. 17, 2-7 (2018).

25. Bock, C. et al. Improved morphology and charge carrier injection in pentacene field-effect transistors with thiol-treated electrodes. J. Appl. Phys. 100, 114517 (2006).

26. Shree, S. et al. High optical quality of $\mathrm{MoS}_{2}$ monolayers grown by chemical vapor deposition. 2D Mater. 7, 015011 (2019).

27. George, A. et al. Controlled growth of transition metal dichalcogenide monolayers using Knudsen-type effusion cells for the precursors. J. Phys. Mater. 2, 016001 (2019).

28. Zaumseil, J. \& Sirringhaus, H. Electron and ambipolar transport in organic fieldeffect transistors. Chem. Rev. 107, 1296-1323 (2007).

29. Zschieschang, U. et al. Roadmap to gigahertz organic transistors. Adv. Funct. Mater. 30, 1903812 (2020).

30. Werkmeister, F. \& Nickel, B. Towards flexible organic thin film transistors (OTFTs) for biosensing. J. Mater. Chem. B 1, 3830-3835 (2013).

31. Ramuz, M., Tee, B. C. K., Tok, J. B. H. \& Bao, Z. Transparent, optical, pressuresensitive artificial skin for large-area stretchable electronics. Adv. Mater. 24, 3223-3227 (2012).

\section{ACKNOWLEDGEMENTS}

B.N. and S.B.K. acknowledge Philipp Altpeter (LMU) for cleanroom support, Domenikos Chryssikos (TUM) for the SAM modification recipe, Joachim Rädler (LMU) for comments on wetting physics and Michel de Jong (UT) for ALD grown alumina wafer. All authors acknowledge financial support by EU within FLAG-ERA JTC 2017 managed Deutsche Forschungsgemeinschaft (DFG) under contract nr. NI 632/61 and TU 149/9-1. B.N. acknowledges support from the Bavarian State Ministry of Science, Research and Arts through the grant "Solar Technologies go Hybrid (SolTech)".

\section{AUTHOR CONTRIBUTIONS}

The research project was initiated by B.N. and A.T. S.B.K., A.G., A.T., and B.N. developed the OSC transfer technique. S.B.K. pursued the growth and transfer of organic nanosheets including their characterization with help from F.A. E.N., Z.G. and A.G. carried out the preparation of TMDs. S.B.K. and U.H. fabricated OSC/TMD devices. S.B.K. characterized the unipolar and ambipolar FETs while E.N. and A.G. measured the antiambipolar FET. S.B.K. and B.N. analyzed the FET characteristics and wrote the manuscript. All authors contributed to the final form of the manuscript.

\section{FUNDING}

Open Access funding enabled and organized by Projekt DEAL.

\section{COMPETING INTERESTS}

An institutional patent application titled "Method of transfer of organic semiconductor films to a substrate and electronic devices made therefrom" with the German Patent and Trade Mark Office (GPTO) was filed by FSU Jena with the official file number "10 2021107 057.0." Inventors (share equal part, in alphabetic order): Antony George, Sirri Batuhan Kalkan, Bert Nickel, Andrey Turchanin.

\section{ADDITIONAL INFORMATION}

Supplementary information The online version contains supplementary material available at https://doi.org/10.1038/s41699-021-00270-9.

Correspondence and requests for materials should be addressed to Andrey Turchanin or Bert Nickel.

Reprints and permission information is available at http://www.nature.com/ reprints

Publisher's note Springer Nature remains neutral with regard to jurisdictional claims in published maps and institutional affiliations.

Open Access This article is licensed under a Creative Commons Attribution 4.0 International License, which permits use, sharing, adaptation, distribution and reproduction in any medium or format, as long as you give appropriate credit to the original author(s) and the source, provide a link to the Creative Commons license, and indicate if changes were made. The images or other third party material in this article are included in the article's Creative Commons license, unless indicated otherwise in a credit line to the material. If material is not included in the article's Creative Commons license and your intended use is not permitted by statutory regulation or exceeds the permitted use, you will need to obtain permission directly from the copyright holder. To view a copy of this license, visit http://creativecommons. org/licenses/by/4.0/.

(c) The Author(s) 2021 